\title{
Development of PVC films, resistant mold influence
}

\author{
Aliya Mazitova*, Albina Maskova ${ }^{\dagger}$ and Guliya Aminova \\ Ufa State Petroleum Technological University, Mendeleev st., Ufa, 450080, Bashkortostan Republic, \\ Russia
}

\begin{abstract}
Along with the use of plasticizers in the development of polymeric materials with specific properties, special substances such as fungicides are also used because of the negative impact on the polyvinyl chloride materials of microorganisms.

In the present work, the possibility of practical application of butylbutoxyethyl phthalate (BBOEP) and dibutoxyethyl adipate (DBOEA) as plasticizers, and 4-amino-1,4,5,6-tetrahydro-1,2,4-triazinone-5 as a fungicide, in PVC films. The first additive was obtained by the esterification of phthalic anhydride (adipic acid) with hydroxyethyllylated butanol. The second additive was obtained on a base of ethyl monochloroacetate.

According to the conducted experiments, it was it was found that when exposed to mold fungi, some physic-mechanical characteristics of plastic compounds deteriorate.

During the study of the resistance to fungi of plastic compounds, it was found that compositions made on the basis of PVC with BBOEP have got high funginertness, while compositions prepared with using of DBOEA turned out to be unstable against to microorganisms. For the prevention infection by the fungus of the obtained plastic compounds based on adipic acid, a proposed fungicide was used. Primary studies have shown that DBOEA with the addition of the fungicide for resistance to fungi is not inferior to BBOEF.
\end{abstract}

\section{Introduction}

To date, due to existence of a number of unique physic - mechanical, operational and strength properties polymers have no analogs among traditional constructional materials. Polymeric materials are very popular both in industry and in everyday life, as evidenced by the steady increase in the rate of their production and expand their areas of application. The wide application of polyvinyl chloride is primarily connected with the availability of raw materials, relatively low cost and the possibility of obtaining on its basis materials with

\footnotetext{
* Corresponding author: elenaasf@yandex.ru

† Corresponding author: asunasf@ mail.ru

† Corresponding author: aminovagk@inbox.ru
} 
different performance characteristics that correspond virtually any consumer requirements. On the other side, PVC has got an original complex of properties: high enough strength, good dielectric, as well as electrical and thermal insulation properties. The advantages of PVC include high resistance to aging and oxidation, to the action of lubricating oils and hydroxides. For this reason today polyvinyl chloride is widely used in medicine, technology, in agriculture, as well as in the construction sector and in everyday life $[1,2]$.

Pure polyvinyl chloride is difficult to process, so it is mixed with various additives that expand the field of application of PVC and allow to consider it as a multicomponent system and the most easily blendable thermoplastic polymer. The choice of the type and dosage of the initial components is determined by the conditions of processing the polymer composition and the necessary complex of operational properties of the products obtained. The choice of the type and dosage of the initial components is determined by the conditions of processing the polymer composition and the necessary complex of operational properties of the products obtained. The content of additives in the polymer composition may vary within very wide limits. Depending on the task, the type and nature of the polymer additives, it can range from one percent to $95 \%[2,3]$.

A lot of works of domestic and foreign scientists are devoted to the development of composite materials based on PVC: V.A. Voskresenskiy, B.P. Shtarkman, K.S. Minsker, R.S. Barstein, F. Grossman, J. Summers, C. Wilkie, C. Daniels, C. Tinius, O.A. Legonkova, E.A. Pekhtasheva, I.G. Kanevskaya, V.D. Ilyichev et al. Fundamental researches have allowed to develop the basic principles of the formulation of PVC compositions, to estimate the effect of plasticizers and other additives on the properties of polymeric materials [2-11]. One of the ways to improve the physico - mechanical properties of PVC materials and simplification the processing of the polymer into the corresponding products is plasticization $[3,12]$.

In the industry, the most popular plasticizers of PVC are esters. The volume of their production accounts for $85 \%$ of the total output of all plasticizers. Esters of phthalic (phthalates), phosphoric (phosphates), sebacic (sebacates), and adipic acids (adipates) are of the greatest practical interest [3].

In adverse conditions, plasticized PVC materials can be exposed to various microorganisms, mainly mold, which adversely affects on appearance of plastic compounds (deterioration of decorative and other external qualities due to the appearance of mold stains) and reduces their service life as a result of changes in some physical and mechanical properties of material due to condensation of water vapor from the atmosphere or accumulation of moisture.

In some cases, the problem of resistance of plasticized polymers to the action of mold is considered as a problem of the resistance of the plasticizer, because some types of mold use plasticizers, that are included in the compositions, as a power source and accelerate the aging process of plastics. Therefore from the resistance of the plasticizer to biological corrosion to a large extent depends on the biostability of the entire material.

Literature data analysis indicates that orthophthalic acid esters plasticizers are more resistant to mold fungi than esters of aliphatic acids [3, 8-11].

One of the way of protection of plasticized PVC materials from negative influence of mold fungi is introduction of fungicides in compositions. Nitrogen containing heterocyclic compoundsare widely reported in the publications. The streamlined synthesis of compounds with wide spectrum of essential properties is prospective lines of development of chemistry of nitrogen containing heterocyclic compounds. Triazines hold a specific place among the great variety of nitrogen containing heterocyclic compounds. Derivatives of unsymmetrical triazines demonstrate a large range of bioactive action, they were offered in the quality of herbicides, plant growth regulators, insecticides, antifungal, acaricide, pharmaceutical and 
veterinary preparations, stabilizer-antioxygen of polymers and also as corrosion inhibitor [13-18].

In this work the results of an investigation of new methods of synthesis and of the chemical-physical properties of butylbutoxyethyl phthalate and dibutoxyethyl adipate, as well as derivatives of 1,2,4-triazine's with aminogroup in 4- positions are shown.

\section{Experimental part}

For the development of new materials that meet the increased modern requirements of operation, we have obtained plasticizers - butylbutoxyethyl phthalate (BBOEP) and dibutoxyethyl adipate (DBOEA), and a fungicide - 4-amino-1,4,5,6-tetrahydro-1,2,4triazinone- 5 .

Butylbutoxyethyl phthalate and dibutoxyethyl adipate were prepared in two steps.

First of all ethoxylated butanol was synthesized. In a four-necked flask equipped with a stirrer, a thermometer, a reflux condenser, and a device for introducing ethylene oxide, the calculated amount of butanol and the catalyst - caustic soda were loaded. The reactor was heated in an oil bath and blowed with nitrogen to remove air. Then gradually (with the mixer running), ethylene oxide was introduced. The rate of ethylene oxide feeding was adjusted so that the unreacted oxide condenses in the reflux condenser and flows back into the reactor "without liquid flooding". After feeding of ethylene oxide, the reaction mixture was further heated and then cooled to room temperature. The catalyst was neutralized with a calculated amount of sulfuric acid and the resulting mass was filtered.

At the next step, by esterifying it with phthalic anhydride (adipic acid), the final products were obtained. In this regard, phthalic anhydride (adipic acid), ethoxylated butanol and the catalyst, n-toluenesulfonic acid (PTSA) (tetrabutoxytitanium) were loaded into a three-necked flask equipped with refrigerator with the Dean-Stark trap, thermometer and mechanical stirrer. Activated carbon was used when required. At the and of the reaction, the catalyst was hydrolyzed with water and the product of esterification was filtered off. In case of using PTSA, an inert gas - carbon dioxide or nitrogen - was bubbled through the reaction mixture to facilitate the removal of water that forms.

The maximum yield of plasticizers $(87-89 \%)$ was achieved under the following conditions: the butanol: ethylene oxide molar ratio was $1: 1$, the amount of catalyst was 0.5 $-3 \%$ (wt. of loading), and the temperature was $110-140{ }^{\circ} \mathrm{C}$; the molar ratio of phthalic anhydride (adipic acid): ethoxylated butanol $-1: 2.5$, the amount of catalyst $0.1-2 \%$ (wt. from loading) + activated carbon in an amount of $1 \%$ (wt. from the mass of loaded components), temperature $-110-170^{\circ} \mathrm{C}$ [19-24].

The general scheme for obtaining plasticizers has got the following form: 

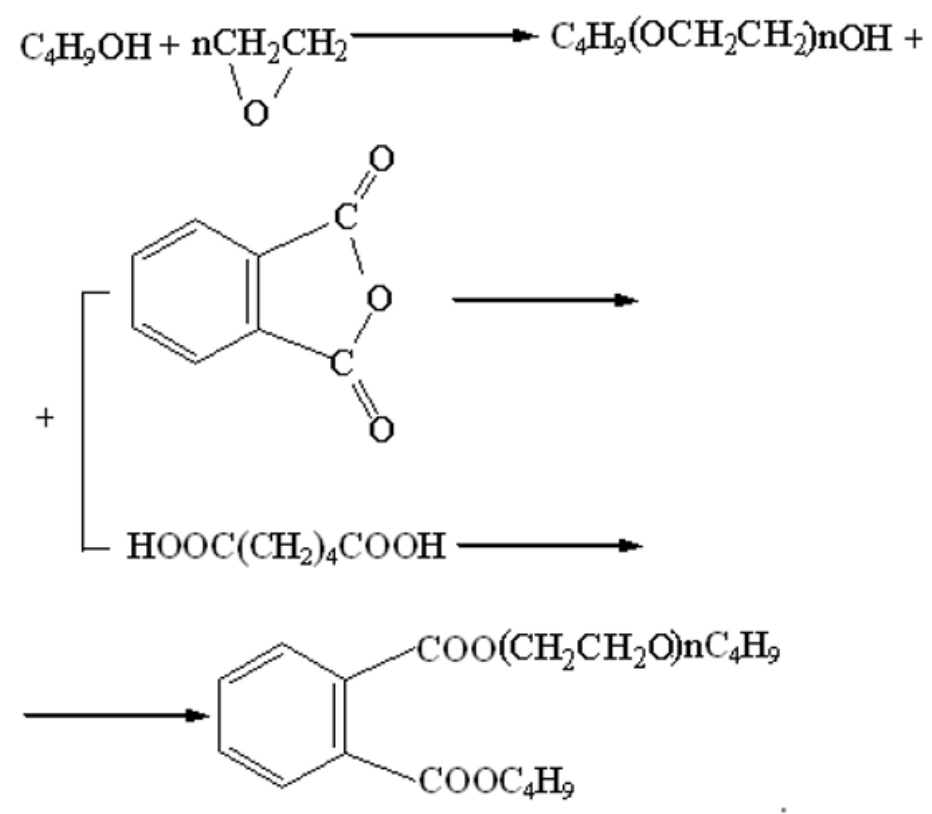

1

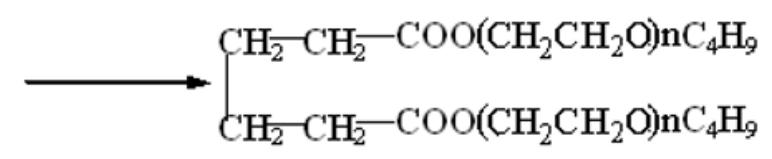

2

Physic-chemical properties of obtained esters are given in Table 1.

Table 1. Physico - chemical properties of plasticizers.

\begin{tabular}{|l|c|c|}
\hline \multicolumn{1}{|c|}{ Characteristics } & \multicolumn{2}{|c|}{ № compound } \\
\cline { 2 - 3 } & $\mathbf{1}$ & $\mathbf{2}$ \\
\hline Yield, \% & 89 & 87 \\
\hline Ester number, $\mathrm{mg} \mathrm{KOH} / \mathrm{g}$ & 345 & 325 \\
\hline Density at $20^{\circ} \mathrm{C}, \mathrm{g} / \mathrm{sm}^{3} \mathrm{~d}^{20}{ }_{4}$ & 0.901 & 0.994 \\
\hline Acid number, $\mathrm{mg} \mathrm{KOH} / \mathrm{g}$ & 0.10 & 0.08 \\
\hline
\end{tabular}

Physic-chemical characteristics of plasticizers were analyzed according to GOST 8728 88.

4-amino-1,4,5,6-tetrahydro-1,2,4-triazinon-5 was obtained in several steps.

In the first stage, hydrazinoacetic acid ethyl ester was synthesized by mixing of monochloroacetic acid (MCAA) with an excess of hydrazine hydrate. MCAA was added to the aqueous solution of hydrazine in several stages. Further sodium hydroxide was added and the reaction mixture was evaporated under vacuum. An alcoholic solution of hydrogen chloride was added to the dry residue and the mixture was carefully heated to boiling. Then the mixture was cooled and saturated with hydrogen chloride gas, alcohol was added, heated to boiling and the hot solution was filtered.

When the solution is cooled, the hydrochloride of hydrazino ester crystallizes. 
At the next stage hydrazide of hydrazinoacetic acid was obtained by the reaction of hydrazine hydrate with ethyl ester of hydrazinoacetic acid. In a three-necked flask equipped with a mechanical stirrer, hydrazine hydrate, the solvent - dimethylformamide (DMFA) were placed, and the hydrazinoacetic acid ethyl ester in DMFA was gradually added. The reaction mixture was hold at room temperature. The precipitate was filtered off, washed with hexane and recrystallized from alcohol.

At the last stage of the synthesis, hydrazinoacetic acid hydrazide was cyclized to the target triazine. In a flask equipped with a mechanical stirrer, hydrazinoacetic acid hydrazide was placed, formic acid was added and heated until dissolved. The reaction mixture was boiled for three hours until the formic acid removal. Water was added and evaporated to dryness. The dry residue was washed with acetone and dried in air.

The maximum yield of the desired 4-amino-1,4,5,6-tetrahydro-1,2,4-triazinone-5, in conversion to the starting material, is achieved under the following conditions: in the first stage, hydrazinoacetic acid ethyl ester is synthesized with a yield of $86 \%$ at a temperature $80^{\circ} \mathrm{C}$ and molar ratio MCAA: hydrazine hydrate 1: 5; on the next stage - hydrazinoacetic acid hydrazide ( $83 \%$ ) by carrying out the reaction in DMF at a temperature of $20-25^{\circ} \mathrm{C}$ during one hour; on the last stage - 4-amino-1,4,5,6-tetrahydro-1,2,4-triazinon-5 (93\%) by carrying out the reaction with the ratio of the initial reagents hydrazinoacetic acid hydrazide: formic acid $1: 2,5$ for three hours at a temperature of $105^{\circ} \mathrm{C}$ [13-18].

The general scheme for obtaining a fungicide has got the following form:
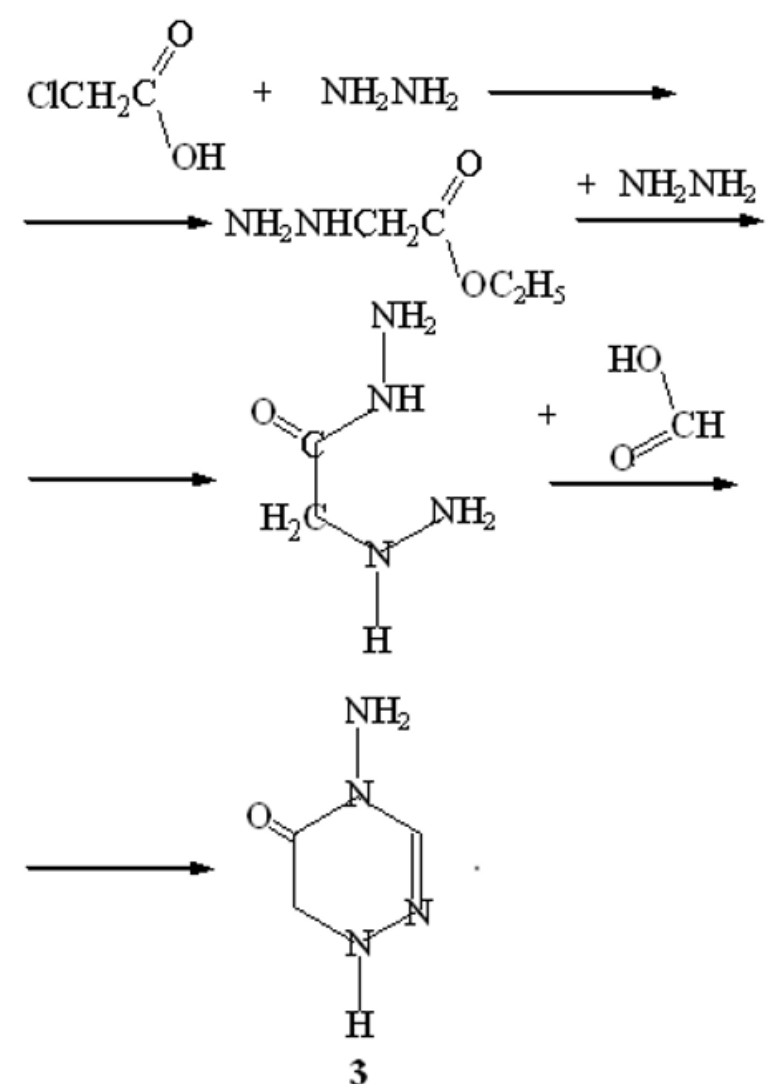

The physic - chemical and spectral characteristics of 4-amino-1,4,5,6-tetrahydro-1,2,4triazinone- 5 are given in Table 2 . 
Table 2. Physic - chemical and spectral characteristics of fungicide.

\begin{tabular}{|c|c|c|}
\hline \multirow{2}{*}{\multicolumn{2}{|c|}{ Characteristics }} & № compound \\
\hline & & 3 \\
\hline \multicolumn{2}{|l|}{ Yield, \% } & 93 \\
\hline \multicolumn{2}{|l|}{ Melting point, ${ }^{\circ} \mathrm{C}$} & $149-151$ \\
\hline \multicolumn{2}{|l|}{ IR - spectrum, sm $^{-1}$} & $3400,1460,1630$ \\
\hline \multicolumn{2}{|c|}{ Mass - spectrum, m/e } & $113,71,42$ \\
\hline \multirow{2}{*}{ Elemental analysis } & Found N, \% & 49.96 \\
\hline & Calculated N, \% & 49.56 \\
\hline
\end{tabular}

\section{Results and discussion}

Obtained orthophthalic and adipic acid esters were tested in PVC formulations of general purpose films and materials for the insulation of wires and cables. Obtained orthophthalic and adipic acid esters were tested in PVC formulations of general purpose films and materials for the insulation of wires and cables. Films for testing were obtained by rolling of PVC-compositions, previously prepared on a high-speed mixer. In all cases, there were no technological difficulties in the preparation and processing of compositions. Physical and mechanical parameters were determined according to the requirements of current standards: the obtained samples of "General purpose" ("GP") films were analyzed according to GOST 16272-79 "Polyvinyl chloride plasticized technical film", the obtained samples of cable plastic compounds O-40 rec. OM-40 (black) was analyzed according to GOST 5960-72 rev. 1 - 9 "Polyvinyl chloride for insulation and protective sheaths of wires and cables".

Results of testing in the formulation of the film brand "GP" and in the formulation of cable plastic brand O-40 rec. OM-40 (black) are shown in Table 3.

Table 3. Results of testing plasticizers in the PVC - formulation

(Formulation, wt. parts: PVC -100 , plasticizer -42 , stabilizer - calcium stearate -3 ).

\begin{tabular}{|c|c|c|c|c|}
\hline \multirow{2}{*}{\multicolumn{2}{|c|}{ Characteristics }} & \multirow{2}{*}{$\begin{array}{l}\text { Norms according to } \\
\text { GOST }\end{array}$} & \multicolumn{2}{|c|}{ Plasticizer } \\
\hline & & & BBOEP & DBOEA \\
\hline \multicolumn{5}{|c|}{ Testing in the PVC-formulation of film brand "GP" according to GOST16272-79 } \\
\hline \multirow{2}{*}{$\begin{array}{l}\text { Breaking strength, } \\
\mathrm{MPa}\end{array}$} & along & no less than 11.8 & 14.2 & 13.8 \\
\hline & across & no less than 9.8 & 13.5 & 13.2 \\
\hline \multicolumn{2}{|l|}{ Tensile strain, \% } & no less than 140 & 185 & 178 \\
\hline \multicolumn{2}{|l|}{ Shatter point, ${ }^{\circ} \mathrm{C}$} & Not above -25 & \multicolumn{2}{|c|}{ Stand the test } \\
\hline \multicolumn{5}{|c|}{$\begin{array}{l}\text { Testing in the PVC-formulation of cable plastic brand O-40 rec. OM-40 (black) according to GOS } \\
5960-72 \text { rev. 1-9 (first quality) }\end{array}$} \\
\hline \multicolumn{2}{|c|}{ Breaking strength, $\mathrm{MPa}$} & no less than 13.7 & 14.6 & 14.3 \\
\hline \multicolumn{2}{|c|}{ Breaking elongation, $\%$} & no less than 280 & 345 & 330 \\
\hline Shatter point, ${ }^{\circ} \mathrm{C}$ & & Not above -40 & \multicolumn{2}{|c|}{ Stand the test } \\
\hline
\end{tabular}

According to the Table 3, testing of plasticizers in the industrial PVC formulations of "GP" films and O-40 rec plastic cable plastics. OM-40 (black), showed their high efficiency. The obtained PVC films and materials for insulation of wires and cables are not only not inferior in terms of the requirements of existing standards, but also exceed them in some physic - mechanical properties: strength and breaking elongation and shatter point are improved. This indicates that the processing of the corresponding PVC compositions is facilitated. 
For investigation of the effect of the obtained plasticizers on the resistance to biocorrosion, testing of PVC-plastic compounds in the soil for a certain period were carried, after which several physical and mechanical parameters were determined. Results of testing are shown in Table 4.

Table 4. Results of testing plasticizers in the PVC - formulation after aging in soil

(Formulation, wt. parts: PVC -100 , plasticizer -42 , stabilizer - calcium stearate -3 ).

\begin{tabular}{|c|c|c|c|c|}
\hline \multirow{2}{*}{\multicolumn{2}{|c|}{ Characteristics }} & \multirow{2}{*}{$\begin{array}{c}\text { Norms according to } \\
\text { GOST }\end{array}$} & \multicolumn{2}{|c|}{ Plasticizer } \\
\hline & & & BВОЕР & DBOEA \\
\hline \multicolumn{5}{|c|}{ Testing in the PVC-formulation of film brand "GP" according to GOST16272-79 } \\
\hline \multirow{2}{*}{$\begin{array}{l}\text { Breaking strength, } \\
\mathrm{MPa}\end{array}$} & along & no less than 11.8 & 7.8 & 6,8 \\
\hline & across & no less than 9.8 & 5.7 & 4,5 \\
\hline \multicolumn{2}{|l|}{ Tensile strain, \% } & no less than 140 & 120 & 117 \\
\hline \multicolumn{2}{|l|}{ Shatter point, ${ }^{\circ} \mathrm{C}$} & Not above -25 & \multicolumn{2}{|c|}{ Doesn't stand the test } \\
\hline \multicolumn{2}{|c|}{ Resistance to fungi, points } & Not resistant & resistant & Not resistant \\
\hline \multicolumn{5}{|c|}{$\begin{array}{l}\text { Testing in the PVC-formulation of cable plastic brand O-40 rec. OM-40 (black) according to GOST } \\
5960-72 \text { rev. } 1 \text { - } 9 \text { (first quality) }\end{array}$} \\
\hline \multicolumn{2}{|c|}{ Breaking strength, MPa } & no less than 13.7 & 10.6 & 9.3 \\
\hline \multicolumn{2}{|c|}{$\begin{array}{l}\text { Breaking elongation, \% } \\
\text { Shatter point }{ }^{\circ} \mathrm{C}\end{array}$} & no less than 280 & 260 & 254 \\
\hline Shatter point, ${ }^{\circ} \mathrm{C}$ & & Not above -40 & \multicolumn{2}{|c|}{ Doesn't stand the test } \\
\hline \multicolumn{2}{|c|}{ Resistance to fungi, points } & Not resistant & resistant & Not resistant \\
\hline
\end{tabular}

From the data Table 4 it is apparent that under the mold fungi inflience, the strength and breaking elongation are deteriorated and the brittleness somewhat increases, the specific surface electrical resistance decreases (Fig. 1), which is probably connect with the absorption of fungi by the films.

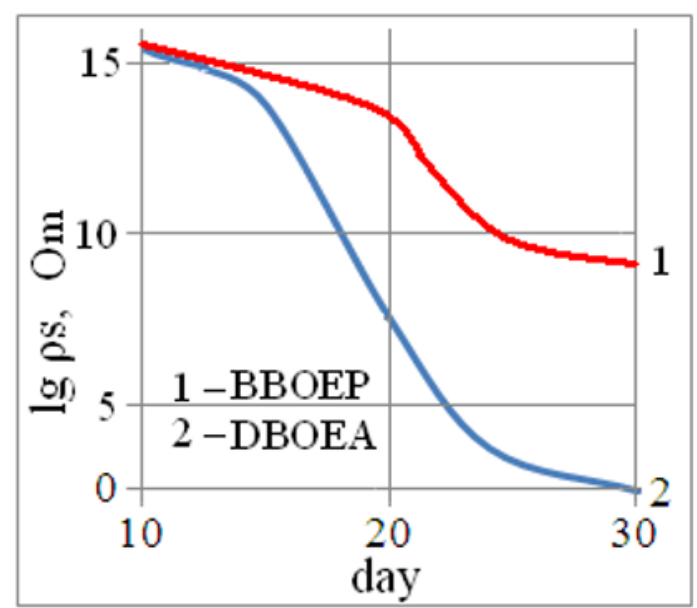

Fig. 1. The change in specific surface resistance from the duration of influence of mold fungi.

During the study of the fungi resistance of plastics, it was found that compositions made on the basis of PVC with butylbutoxyethyl phthalate have got a high mushroom resistance, while compositions prepared using dibutoxyethyl adipate turned out to be unstable with respect to microorganisms.

To prevent infection by the fungus in the resulting plastics, a 4-amino-1,4,5,6tetrahydro-1,2,4-triazinone-5 as fungicide was used. Primary studies have shown that 
DBOEA with the addition of the proposed fungicide to protect materials from damage by fungi (mainly mold) in the PVC-compositions in the amount of $2 \%$ in fungi resistance is not inferior to BBOEP.

\section{Conclusions}

In such way when in industrial PVC formulations of "GP" films and O-40 rec plastic cable plastics. OM-40 (black) developed plasticizers are used, their main physical and mechanical properties: "Breaking strength", "Tensile strain" and "Shatter point" are improved.

Under influence of mold fungi on plasticized PVC materials, the strength and breaking elongation are deteriorated and brittleness somewhat increases, the specific surface electrical resistance decreases, probably due to the destruction of the plasticizer by mold fungi. It should be noted that the PVC compositions on the basis of DBOEA in the process of aging in the soil showed poor fungi resistance.

To prevent infection by the fungus in the resulting plastics, a 4-amino-1,4,5,6tetrahydro-1,2,4-triazinone-5 as fungicide was used. Preliminary studies have shown that DBOEA with the addition of the proposed fungicide in the amount of $2 \%$ for fungi resistance is not inferior to BBOEP.

\section{References}

1. M. Hebole, K. Tomus, Mach. Des., 57, 8, 164-165 (1987)

2. Ch. Uilki, J. Sammers, Ch. Daniels, Polyvinylchloride (Profession, Saint-Petersburg, 2007)

3. R.S. Barshteyn, V.I. Kirilovich, Y.E. Nosovskiy, Plasticizers for polymer. (Chemistry, Moscow, 1982)

4. V.A. Voskresenskyi, E.M. Orlova, E.I. Abramova, N.S. Prohorova, Rus. Chem. Review, 15, 1, 142-159 (1971)

5. K.S. Minsker, G.T. Fedoseeva, Degradation and stabilization of polyvinylchloride (Chemistry, Moskow, 1979)

6. B.P. Shtarkman, PVC plasticizer. (Chemistry, Moscow, 1975)

7. F. Grossman, Guidline for development of PVC compositions (Scientific foundation and technologies, Moscow, 2009)

8. O.A. Legonkova, L.A. Sukhareva, One thousand and one polymer is biostable to biodegradable (RadioSoft, Moscow, 2004)

9. E.A. Pekhtasheva, Biological damage and protection of non-food of goods: a textbook for bachelors (Dashkov and Co., Moscow, 2012)

10. I.G. Kanevskaya, Biological damage to industrial materials (Science, Leningrad, 1984).

11. V.D. Ilyichev, Biological damage (Higher school, Moscow, 1987)

12.P.V. Kozlov, S.P. Popkov, Physico-chemical basis of plasticization of polymers (Chemistry, Moscow, 1982).

13. A.K. Mazitova, V.H. Khamaev, A.S. Ulyamaeva, I.A. Sukhareva, Bash. Chem. J, 6, 4, 4-9 (1999)

14. A.K. Mazitova, V.H. Khamaev, Unbalanced triazine. Synthesis, properties and application (Reaktiv, Ufa, 1999)

15. A.K. Mazitova, G.F. Aminova, I.A.Sukhareva, D.R. Galieva, Bash. Chem. J., 14, 2, $25-$ 29 (2007)

16. S.R. Faizullina, E.A. Kalistratova, E.A. Builova, D.R. Galieva, A.K. Mazitova, Bash. Chem.. J., 19, 3, 92-94 (2012) 
17. S.R. Faizullina, D.R. Sadykova, M.S. Klyavlin, D.U. Rysaev, A.K. Mazitova, Elect. Sci. J. «Oil and gas business», 6, 552-562 (2013)

18. A.K. Mazitova, I.A. Sukhareva, E.A. Builova, L.Z. Rolnik, Sh.T. Aznabaev, A.F. Aminova, Bash. Chem. J., 21, 3, 64-68 (2014)

19. A.K. Mazitova, G.F. Aminova, A.I. Gabitov, A.R. Maskova, B.R. Khusnutdinov, A.M. Fattakhova, Elect. Sci. J. «Oil and gas business», 12-1, 120-127 (2014)

20. A.K. Mazitova, L.B. Stepanova, G.F. Aminova, A.R. Maskova, Ind. Prod. and use of Elast., 2, 27-31 (2015)

21. A.K. Mazitova, G.K. Aminova, A.I. Gabitov, A.R. Maskova, R.G. Rahmatullina. Bash. Chem. J., 22, 3, 23-26 (2015)

22. G.F. Faizullina, A.I. Gabitov, A.R. Maskova, I.I. Ahmetova. Oil and gas business, 15, 3, 106-111 (2017)

23. A.K. Mazitova, G.K. Aminova, A.R. Maskova, G.G. Yagafarova, R.M. Mazitov, Nanotechnologies in Construction, 9, 4, 48-63 (2017) 24. A.K. Mazitova, G.K. Aminova, A.R. Maskova, I.N. Sabitov, I.V. Nedoseko, Nanotechnologies in Construction, 9, 6, 168-180 (2017) 